\title{
Correction to: Construction Safety Informatics
}

\section{Correction to:}

R. Y. M. Li, Construction Safety Informatics, https://doi.org/10.1007/978-981-13-5761-9

The original version of the Acknowledgement section in the book front matter has been revised.

1. The numbering of chapters contributed by co-author Wilson Weisheng Lu has been changed from " 2,6 and 7" to " 2,4 and 6".

2. The affiliation of co-author Daniel Chi Wing Ho has been changed to "Technological and Higher Education Institute of Hong Kong".

3. Funding information has been added. 